\title{
Uso de Facebook, estrés percibido y consumo de alcohol en jóvenes universitarios
}

\author{
Use of Facebook, perceived stress and alcohol consumption \\ among university students
}

Edna Idalia Paulina Navarro Oliva ${ }^{1}$

Edilaine Cristina da Silva Gherardi-Donato ${ }^{2}$

Javier Álvarez Bermúdez ${ }^{3}$

Francisco Rafael Guzmán Facundo ${ }^{4}$

${ }^{1}$ Facultad de Enfermería Dr. Santiago Valdés Galindo, Universidad Autónoma de Coahuila. Calz Francisco I. Madero 1237, Zona Centro. 25000 Saltillo Coah. México.

eipno@yahoo.com.mx ${ }^{2}$ Escola de

Enfermagem de Ribeirão Preto, Universidade de São Paulo. Ribeirão Preto SP Brasil.

${ }^{3}$ Facultad de Psicología, Universidad Autónoma de Nuevo León (UANL). San Nicolás de los Garza NL México.

${ }^{4}$ Facultad de Enfermería, UANL. Garza NL México.

\begin{abstract}
Analysis of the effect between the use of Facebook, perceived stress and alcohol consumption among young people. A descriptive, correlative and cross-sectional study was carried out. The sample of 1110 young university students from Nuevo León, Mexico, was selected by probability sample. Personal Data and Prevalence of Alcohol Consumption Inventory, Alcohol Use Disorders Identification Test (AUDIT), Facebook Use Identification Test, Perceived Stress Scale were used. The Research Ethics Committee approved it. We observed $6.4 \%$ of young people have mentioned that the use of Facebook affected the alcohol consumption. Seeing adds with alcohol on Facebook was related to alcohol consumption ( $r s=0,204$, $p<0,05)$. At the time Facebook was used, it was related to the perceived stress ( $r s=0,189, p<$ 0,05). Finally, it was observed that there is a significant impact between the hours and days of use of Facebook, the stress perceived by young people, the age and gender regarding the harmful alcohol consumption among university students ( $R 2$ $=30,9 \%, p=0,003)$. Therefore, it is crucial and necessary to consider social networks an important variable to be included in future interventions regarding mental health.
\end{abstract}

Key words Social network, Stress, Consume alcohol, Young
Resumen Analizar el efecto entre el uso de Facebook, el estrés percibido y el consumo de alcohol en jóvenes. Fue realizado un estudio descriptivo, correlacional y transversal. La muestra fue de 110 jóvenes universitarios jóvenes universitarios de Nuevo León, México. Muestreo probabilístico. Se utilizó una Cedula de Datos Personales y Prevalencias del Consumo de Alcohol, Cuestionario de Identificación de Trastornos por Uso del Alcohol (AUDIT), Cuestionario de Uso de Facebook y la Escala de Estrés Percibido. Se contó con la aprobación del Comité de Ética en Investigación. Se encontró que el 6,4\% de los jóvenes mencionaron que el uso de Facebook ha afectado su consumo de alcohol. El visualizar anuncios con contenido de alcohol en Facebook se relacionó con el consumo de alcohol $\left(r_{s}=0,204, p<0,05\right)$. Las horas que se utiliza Facebook se relacionó con el estrés percibido $\left(r_{s}=0,189, p<0,05\right)$. Finalmente se observó que existe un efecto significativo entre las horas $y$ días de uso de Facebook, el estrés percibido por los jóvenes, la edad y el sexo sobre el consumo de alcohol perjudicial en los jóvenes universitarios $\left(R^{2}=\right.$ $30,9 \%, p=0,003)$. Por lo tanto es importante $y$ necesario el considerar las redes sociales como una variable importante para ser incluida en futuras intervenciones en salud mental.

Palabras clave Red social, Estrés, Consume de alcohol, Jóvenes 


\section{Introducción}

Los jóvenes universitarios se han situado como una de las poblaciones más vulnerables a desarrollar adicción al consumo de alcohol, se menciona que al consumir este tipo de droga lícita aumenta ocho veces más las posibilidades de iniciar el consumo de otras drogas ${ }^{1}$. En el mundo aproximadamente cuatro de cada cinco jóvenes consumen alcohol y más del $40 \%$ de ellos reportan participar en borracheras ${ }^{2}$.

En México, el consumo de alcohol en jóvenes pasó del 40 al $70 \%$ y el patrón de consumo consiste en beber grandes cantidades de alcohol en fiestas o reuniones hasta llegar a la embriaguez, frecuentemente durante los fines de semana. Este patrón de consumo de los jóvenes mexicanos integra dos los factores para llegar rápidamente a la dependencia: la regularidad de uso y abuso en la cantidad de alcohol ${ }^{3}$.

En este sentido, uno de los tipos de consumo de alcohol que actualmente ha ido en aumento entre la población joven mexicana es el consumo de alcohol perjudicial o dañino, el cual crea diversas consecuencias a la salud física, mental y la imagen social de los jóvenes, se considera consumo perjudicial o dañino al consumir en un día típico 10 o más bebidas estándar en los hombres y 6 o más bebidas estándar en las mujeres ${ }^{3,4}$.

Es importante resaltar que el consumo de alcohol se caracteriza por ser un fenómeno multicausal, dentro de los factores que se han identificado se encuentran la predisposición genética, las características individuales, los factores sociales, económicos y ambientales ${ }^{5}$. El estrés percibido se encuentra dentro de dichos factores que pueden aumentar el riesgo de consumir alcohol y otras drogas ${ }^{6}$, específicamente en los jóvenes el estrés es común en consecuencia de los múltiples cambios experimentados en esta etapa de edad, además de las diversas situaciones de la vida diaria relacionadas con la escuela, relaciones interpersonales o el trabajo ${ }^{6-8}$.

Como ya se ha mencionado los jóvenes transitan por una etapa considerada como difícil en el desarrollo humano, aunado a los cambios en la dinámica de la sociedad que pueden repercutir considerablemente en esta población. Un ejemplo de estos cambios son la forma en la cual las personas socializan con sus iguales, los jóvenes actualmente se comunican con amigos o familiares a través de sitios que cada vez son más populares e impersonales, como el Facebook, este sitio de socialización ha logrado posicionarse como una de las redes sociales más utilizadas entre los jóvenes donde se destaca que existen aproximadamente 1,320 millones de usuarios activos de Facebook en todo el mundo ${ }^{9}$ y en México se estima que el $88,2 \%$ de los jóvenes utilizan diariamente esta red social ${ }^{10}$.

Es importante mencionar que aún no es claro como el uso de Facebook puede beneficiar o repercutir en la vida de las persona, ya que mientras para algunos esta plataforma de socialización puede aumentar su satisfacción con la vida y el apoyo social percibido, para otras personas puede ser un factor desencadenante de estrés, ansiedad, depresión, de inicio en el consumo de sustancias o aumento en el patrón de consumo de alcohol, etc ${ }^{6-8}$.

La Asociación Americana de Psicología ${ }^{11}$ menciona que el 39\% de las personas entre 18 y 33 años se han declarado como personas que sufren estrés, así mismo se menciona que los jóvenes que experimentan estrés son más propensos a presentar cuadros de irritabilidad. Se explica que esta población busca estrategias de afrontamiento para liberar las tensiones que provoca el estrés realizando conductas consideradas de riesgo y además que el estrés percibido se ha demostrado que funge como un predictor para el consumo de alcohol ${ }^{6-8}$.

Por todo lo explicado anteriormente se puede observar como el hacer uso de los nuevos espacios virtuales han repercutido en la dinámica social actual, por medio de estos espacios se facilita el estar presente en una plataforma de interacción y organización social, ya que gracias al ritmo de vida tan acelerado que vive actualmente la sociedad es imposible el estar de forma presencial ${ }^{12,13}$. Se menciona que los jóvenes al estar inmersos en estas nuevas formas de interacción y organización pueden experimentar situaciones que se tornan estresantes, ejemplos de estas situaciones son el recibir invitaciones a eventos, el que una publicación o fotografía no sea aceptada por sus amigos mediante los "me gusta" o el contar con un gran número de amigos en esta red social y sientan la responsabilidad de estar pendiente de la actividad mostrada en Facebook ${ }^{13-15}$.

En este sentido, una consecuencia que podrían presentar los jóvenes es el consumo de alcohol, ya que al observar en las redes sociales que sus amigos o familiares llevan a cabo esta conducta de riesgo, podrían adoptar o aumentar su consumo de alcohol; aunado al efecto que el estrés ejerce en el joven, el cual se puede presentar por diversas situaciones (escolares, personales o laborales) y las mismas del uso de Facebook.

De acuerdo a lo expuesto anteriormente se observa la necesidad continuar estudiando sobre estas variables de forma conjunta en los jóvenes 
al considerarse una población de riesgo, con la finalidad de ampliar el conocimiento sobre el consumo de alcohol en México, conocer más sobre la influencia del uso de Facebook en la salud de las personas, el estrés que presentan los jóvenes y favorecer el cuidado preventivo de enfermería en el consumo de alcohol. Por lo anterior, el objetivo es analizar el efecto entre el uso de Facebook, el estrés percibido y el consumo de alcohol en jóvenes.

\section{Método}

Estudio descriptivo, correlacional y transversal. La población se conformó por jóvenes universitarios de diferentes instituciones educativas de Nuevo León, México. El muestreo fue probabilístico, estratificado por institución educativa con asignación proporcional al tamaño de los estratos. El tamaño de la muestra fue de 110 jóvenes universitarios de 18 a 24 años de edad, de los cuales 60 fueron mujeres y en su mayoría se dedicaban solo a estudiar, se calculó a través del paquete estadístico nQuery Advisor ${ }^{\circledR} 7.0^{16}$, con un nivel de significancia de 0.05 , una potencia del $90 \%$ y una tasa de no respuesta del 5\%.

Consumo de alcohol. Se midió a través de las Prevalencias del Consumo de Alcohol y el Cuestionario de Identificación de Trastornos por Uso del Alcohol (AUDIT) validado en población mexicana, consta de 10 reactivos que examinaron el consumo de alcohol durante los últimos doce meses y sus consecuencias. Los reactivos del 1 al 3 determinaron la cantidad y frecuencia del consumo de alcohol, los reactivos del 4 al 6 evaluaron la posibilidad de que exista dependencia al alcohol y los reactivos 7, 8, 9 y 10 examinaron el consumo dañino. Cada pregunta cuenta con una escala tipo Likert con puntuaciones de 0 a 4.

Se tomaron en cuenta los puntos de corte propuestos por la Organización Mundial de la Salud $^{17}$ los cuales mencionan que una puntuación de 0 indica que no existe el patrón del consumo de alcohol, las puntuaciones de 1 a 7 indican un patrón de consumo de riesgo, mientras que de 8 a 19 puntos se muestra un patrón de consumo perjudicial, así mismo se menciona que las puntuaciones de 20 a 40 se considera como un patrón de consumo perjudicial y posible dependencia. Este instrumento ha mostrado ser un instrumento confiable, ya que se han observado Alphas de Cronbach que oscilan entre 0,64 a $0,92^{4,18}$.

Uso de Facebook. Se midió a través de 5 preguntas, las cuales cuantifican la frecuencia y datos generales de uso de Facebook, es decir la exposición sobre contenidos relacionados con el alcohol en Facebook. El tipo de preguntas utilizadas fueron ¿En promedio cuántos días usas Facebook a la semana?, ¿En promedio cuántas horas usas Facebook en un día?, ¿Cuántas veces en los últimos 30 días has visto fotos en Facebook que involucren alcohol?, ¿Cuántas veces en los últimos 30 días has sido invitado por medio de Facebook a una fiesta que involucre el consumo de alcohol?

Así como ¿En promedio cuántos amigos en total tienes en Facebook?, ¿Comúnmente a través de que dispositivo accedes a Facebook?, ¿Sientes que el uso de Facebook ha afectado tu consumo de alcohol? y ¿Sientes que el uso de Facebook ha afectado a otros amigos en su consumo de alcohol?, cada pregunta corresponde a una variable continua, para las preguntas sobre la exposición a contenidos relacionados con el consumo de alcohol en Facebook se realizó un índice, donde se estableció que a mayor puntaje, mayor es la exposición. Es importante mencionar que las preguntas fueron seleccionadas por expertos en prevención de adicciones para conocer sobre el uso de Facebook que presentan los jóvenes.

Estrés Percibido. Se midió a través de la Escala de Estrés Percibido diseñada por Cohen et al. ${ }^{19}$, validada y adaptada culturalmente para población mexicana por González y Landero ${ }^{20}$. Constituida por 14 ítems con una escala tipo Likert con opciones de respuesta de nunca (0) a muy frecuentemente (4). Se establece que a mayor puntuación, mayor será el estrés percibido por la persona. Es importante resaltar que esta escala ha sido utilizada ampliamente en diversos países mostrando confiabilidad y validez, mostrando Alph.as de Cronbach aceptables con valores de 0,79

Para el procedimiento de recolección de datos se contó con la aprobación del Comité de Investigación y el Comité de Ética Investigación de la Facultad de Enfermería de la Universidad Autónoma de Nuevo León, además se solicitó por escrito la autorización para la realización del estudio a los directivos de cada una de las instituciones educativas, Se solicitó consentimiento informado de los jóvenes, la participación fue voluntaria y la información otorgada fue anónima y confidencial.

Los datos fueron procesados en el programa estadístico Statistical Package for the Social Science (SPSS) para Windows V 22,0. Se utilizó estadística descriptiva para obtener frecuencias, proporciones, medidas de tendencia central y de variabilidad; y estadística inferencial no paramétrica para dar respuesta a los objetivos plantea- 
dos, principalmente se realizó la prueba de U de Mann Whitney para conocer las diferencias de consumo entre los jóvenes que perciben que el Facebook ha afectado su consumo y los que no lo perciben así, así mismo se utilizó la prueba de correlación de Spearman y se realizó un Modelo de Regresión Lineal Múltiple. La consistencia interna de los instrumentos se determinó a través del coeficiente de Confiabilidad Alpha de Cronbach, mostrando resultados aceptables.

\section{Resultados}

Participaron 110 jóvenes universitarios, 58,2\% del sexo femenino y con una media de edad de 20,3 años $(D E=2,08)$. La muestra estuvo conformada predominantemente por jóvenes que se encontraban cursando el primer semestre de facultad (30.9\%), de los cuales sólo el 17.3\% mencionó estudiar y trabajar, y el $80 \%$ refirió que aún vivía con sus padres.

El 88,2\% de los jóvenes han consumido alcohol en el último año y el 65,5\% han consumido en el último mes; en relación a los tipos de consumo de alcohol el 52,7\% presentó un consumo de riesgo, el $24,5 \%$ un consumo perjudicial y el $4,5 \%$ ya presentaba dependencia al alcohol o alcoholismo.

En relación al uso de Facebook (Tabla 1) se encontró que el dispositivo más utilizado para acceder a Facebook fue el celular $(82,7 \%)$, además que el 6,4\% de los jóvenes mencionaron que el uso de Facebook ha afectado su consumo de alcohol y el 72,7\% considera que el uso de Facebook que ha afectado el consumo de alcohol de sus amigos. La media del estrés percibido por los jóvenes fue de 25,09 ( $D E=7,03)$.

En relación a si el uso de Facebook ha afectado el consumo de alcohol de los jóvenes (Tabla 2) se identificaron diferencias significativas de consumo de alcohol reportado respecto a si el joven percibe que el uso de Facebook ha afectado su consumo de alcohol $(U=192,500, p=$ 0,038 ), se observa que los jóvenes universitarios que consideran que el uso de Facebook ha afectado su consumo de alcohol presentan puntajes de consumo de alcohol más altos que los jóvenes que no lo consideran.

Los resultados que responden al objetivo del presente estudio muestran el visualizar anuncios con contenido de alcohol en Facebook $\left(r_{s}=\right.$ $0,204, \mathrm{p}<0,05)$, se relaciona positivamente con el consumo de alcohol de los jóvenes universitarios; lo que indica que al visualizar más anuncios de alcohol en Facebook mayor es la ingesta de alcohol. En relación al estrés percibido, se encontró que las horas que se utiliza Facebook $\left(r_{s}=0,189\right.$, $\mathrm{p}<0,05$ ), se relaciona positivamente con el estrés percibido por los jóvenes universitarios; lo que indica que a mayor número de horas de uso de Facebook mayor es el estrés percibido. Así mismo respecto al efecto del uso de Facebook y el estrés percibido sobre el consumo de alcohol perjudicial de los estudiantes universitarios, se observó que existe un efecto significativo, el cual indica que las horas y días de uso de Facebook, el estrés percibido por los jóvenes, la edad y el sexo influyen $\left(R^{2}=30,9 \%, p=0,003\right)$ en el consumo de alcohol perjudicial en los jóvenes universitarios (Tabla 3).

\section{Discusión y conclusión}

Este estudio permitió analizar el efecto entre el uso de Facebook, el estrés percibido y el consumo de alcohol en jóvenes. Respecto al uso de Facebook se encontró que los jóvenes universitarios utilizan la red social cerca de 7 días a la semana y aproximadamente 4 horas al día, esto difiere con otros estudios realizados principalmente en Estados Unidos que señalan un uso diario entre 30 y 90 minutos al día ${ }^{21-24}$. Estos resultados pueden

Tabla 1. Uso de Facebook.

\begin{tabular}{|c|c|c|c|c|c|c|}
\hline & \multirow{2}{*}{$\mathbf{N}$} & \multirow{2}{*}{$\bar{X}$} & \multirow{2}{*}{ Mdn } & \multirow{2}{*}{$\mathrm{DE}$} & \multicolumn{2}{|c|}{ Valor } \\
\hline & & & & & Mínimo & Máximo \\
\hline Días de uso de Facebook por semana & 110 & 6,65 & 7,0 & 0,84 & 2 & 7 \\
\hline Horas de uso de Facebook por día & & 3,96 & 3,0 & 3,36 & 1 & 20 \\
\hline $\begin{array}{l}\text { Invitaciones en Facebook a una fiesta que involucre } \\
\text { alcohol }\end{array}$ & & 4,04 & 2,5 & 5,09 & 0 & 30 \\
\hline Fotos en Facebook que involucren alcohol & & 16,62 & 10,0 & 29,08 & 0 & 300 \\
\hline Amigos en Facebook & & 760,81 & 600,0 & 638,11 & 90 & 4900 \\
\hline
\end{tabular}


Tabla 2. Consumo de alcohol AUDIT de acuerdo al uso de Facebook.

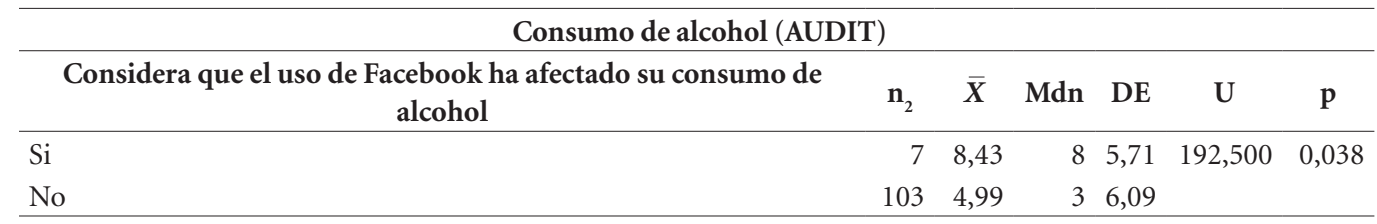

Tabla 3. Influencia del uso de Facebook y el estrés percibido sobre el consumo de alcohol perjudicial de los jóvenes universitarios.

\begin{tabular}{lccccc}
\hline \multicolumn{1}{c}{ Fuente de variación } & SC & Df & CM & F & p \\
\hline Regresión Residual & 6,017 & 15 & 0,401 & 2,618 & 0,003 \\
$\mathrm{R}^{2}=30,9 \%$ & 13,483 & 88 & 0,153 & & \\
\hline \multicolumn{1}{c}{ Modelo $\left(\mathbf{n}_{2}=\mathbf{1 0 3}\right)$} & \multicolumn{7}{c}{ Coeficientes } \\
\cline { 2 - 6 } & 1,814 & 0,792 & & SE & $\mathbf{p}$ \\
\hline (Constante) & $-0,030$ & 0,013 & $-0,240$ & $-2,288$ & 0,025 \\
Horas de uso de Facebook & 0,040 & 0,020 & 0,410 & 2,005 & 0,048 \\
Días de uso de Facebook & 0,029 & 0,014 & 0,217 & 2,157 & 0,034 \\
Estrés percibido & $-0,049$ & 0,021 & $-0,236$ & $-2,328$ & 0,022 \\
Edad & 0,326 & 0,084 & 0,372 & 3,868 & 0,001 \\
Sexo & & &
\end{tabular}

ser explicados por el firme aumento del uso de las nuevas tecnologías entre los jóvenes con la finalidad de mantener vínculos con sus diferentes círculos sociales.

Además se observó que aquellos jóvenes universitarios que mencionaron que el uso de Facebook ha afectado su consumo de alcohol fueron quienes presentaron una media más alta de consumo a diferencia de los jóvenes que no lo mencionaron. Estos resultados concuerdan con otros estudios que mostraron que los jóvenes universitarios consideran que el uso de Facebook ha aumentado su consumo de alcohol ${ }^{6,25,26}$. Estos resultados pueden ser explicados por la influencia que ejercen los pares y crear cierta presión de grupo por realizar conductas de riesgo (consumo de alcohol).

Otro de los hallazgos importantes fue la relación entre la visualización de anuncios con contenido de alcohol y el consumo de alcohol en jóvenes; una explicación de esto es que regularmente los anuncios que son compartidos por las principales compañías de bebidas alcohólicas en Facebook muestran un contexto "positivo" hacía al consumo de alcohol (fiestas, reuniones, antros), las cuales denotan momentos felices especialmente entre los jóvenes. El uso de Facebook como medio de comunicación puede crear una mayor persuasión en el joven que otros medios, ya que al ser compartidas con sus amigos y observadas repetidas veces pueden generar dicha persuasión ${ }^{6,27}$.

Es importante resaltar que la mayoría de los jóvenes pertenecían al sexo femenino, la relación de las mujeres con la herramienta de Facebook contiene particularidades de género. El uso de los medios sociales virtuales es particularmente popular entre las mujeres jóvenes ${ }^{28}$, ellas utilizan más la tecnología para fines de conectividad, a diferencia de los hombres que lo utilizan para medios más egocéntricos ${ }^{29}$.

Evidencias científicas apuntan que las participantes que pasaron más tiempo en Facebook reportaron estar en un estado de ánimo más negativo que las que pasaron menos tiempo en el sitio web de control ${ }^{30}$. Por el diseño metodológico y el objetivo de la presente investigación, los resultados confirman que la cuestión de género merece un abordaje más profundada en la búsqueda de comprensión de construcciones sociales permeadas por las redes sociales que afectarían distintamente hombres y mujeres.

En relación al estrés percibido, se encontró que a mayor número de horas de uso de Face- 
book mayor es el estrés percibido en los jóvenes, estos resultados concuerdan con un reporte realizado en Estados Unidos ${ }^{15}$, donde se menciona que los jóvenes que utilizan Facebook pueden sufrir estrés por el número de amigos o personas agregadas, una explicación a esto es que entre más contactos en esta red social, mayor es el tiempo que se invierte en estar al pendiente de sus actualizaciones, existen más posibilidades de molestarse por algún comentario o tratar de responder a todos los comentarios o mensajes directos, esto aunado a las actividades de la vida diaria $^{15}$.

Estos resultados nos invitan a analizar de una manera más compleja el rol de la tecnología por el uso que se hace. Es conocido que el bienestar está asociado negativamente a estar solo y positivamente al tiempo invertido en conversaciones con otros ${ }^{31}$, el que, teóricamente, ubicaría al Facebook como una herramienta favorable. Sin embargo, el tipo de conversación es un diferencial, en que el alto nivel de bienestar está asociado a más conversaciones substanciales y a pocas pequeñas charlas ${ }^{32}$. Al considerar el tipo de con- versación que se lleva a cabo en las redes sociales, las podemos considerar como charlas superficiales las cuales están negativamente asociadas a la sensación de satisfacción con la vida y bien estar, y explicaría el resultado de la asociación, en este estudio, con el estrés percibido.

Se concluye que existe un efecto entre las horas y días de uso de Facebook, el estrés percibido por los jóvenes, la edad y el sexo sobre el consumo de alcohol (perjudicial) en los jóvenes universitarios. Lo cual es importante hallazgo ya que en México no se cuenta con suficiente información sobre como las nuevas tecnologías están afectando a la salud mental de los jóvenes. Es importante destacar que los resultados presentados son inéditos a nivel mundial y necesitan ser explorados en otros contextos para que se tenga parámetros de comparación de la presentación de las relaciones encontradas en otras realidades socioculturales, además de aportar información importante para el diseño e implementación de futuras intervenciones multiprofesionales que se enfoquen en buscar estrategias que contrarresten los efectos de las nuevas tecnologías en la salud mental.

\section{Colaboradores}

EIP Navarro Oliva - Diseño, investigación, metodología, tratado de los datos y edición final. ECS Gherardi-Donato - Investigación y metodología; FR Guzmán Facundo - Diseño, investigación, edición final; Dr. J Álvarez Bermudez - Investigación y tratado de los datos estadísticos. 


\section{Referencias}

1. Organización Mundial de Salud (OMS). Riesgos para la salud de los jóvenes. 2011. [accesado 2014 Abr 28]. Disponible en: http://www.who.int/mediacentre/factsheets/fs345/es/

2. National Institute on Alcohol Abuse and Alcoholism (NIAAA). Statistical snapshot of college drinking. 2010. [accesado 2012 Abr 27]. Disponible en: http://www. niaaa.nih.gov...StatisticalSnapshotCollegeDrinking. htm

3. Consejo Nacional Contra las Adicciones-Secretaría de Salud (CONADIC-SS). Encuesta Nacional de Adicciones Alcohol. México: CONADIC-SS; 2011.

4. De la Fuente J, Kershenobich D. El alcoholismo como problema médico. Revista Facultad de Medicina UNAM 1992; 35(2):47-51.

5. International Center for Alcohol Policies. Determinants of Drinking. 2009. [accesado 2014 Mar 4]. Disponible en: http://www.icap.org/LinkClick.aspx?fileticket=op SlJDbBI\%2BU\%3D\&tabid=243

6. Egan K, Moreno M. Alcohol References on Undergraduate Males Facebook Profiles. Am J Mens Health 2011; 5(5):413-420.

7. Cooper ML, Rusell M, Skinner JB, Frone MR, Mudar P. Stress and alcohol use: moderating effects of gender, coping and alcohol expectancies. J Abnorm Psychol 1992; 101(1):139-152.

8. Veenstra MY, Lemmens PH, Friesema IH, Tan FE, Garretsen HF, Knottnerus JA, Zwietering PJ. Coping style mediates impact of stress on alcohol use: a prospective population-based study. Addiction 2007; 102(12):18901898.

9. Goodwill Community Foundation. Social media: Facebook 101. 2014. [accesado 2015 Mar 24]. Disponible en: http://www.gcfkearnfree.org/socialmedia/facebook101

10. Instituto Mexicano de la Juventud (IMJUVE). Encuesta Nacional de Juventud. México: IMJUVE; 2010. [accesado 2014 Abr 28]. Disponible en: http://www.imjuventud.gob.mx/pagina.php?pag_id=137

11. American Psychological Association. Los distintos tipos de estrés. 2010. [accesado 2014 Mar 4]. Disponible en: http://www.apa.org/centrodeapoyo/tipos.aspx

12. Peña $A B$. La socialización a través de las redes. REDMARKA 2011; 7:3-24

13. Herrera HM, Pacheco MM, Palomar LJ, Zavala AD. La adicción a Facebook relacionada con la baja autoestima, la depresión y la falta de habilidades sociales. Psicología Iberoamericana 2010; 18(1):6-18

14. Sandin B. El estrés: un análisis basado en el papel de los factores sociales. Revista Internacional de Psicología Clínica y de la Salud 2002; 3(1):141-157.

15. Hampton KN, Rainie L, Lu W, Shin I, Purcell K. Social Media and the Cost of Caring. Washington: Pew Research Center; 2014. [accesado 2014 Mar 4]. Disponible en: http://www.pewinternet.org/2015/01/15/ social-media-and-stress/

16. Elashoff J. nQuery Advisor (Versión 7.0) [Software de computación]. Los Angeles: Statistical Solutions LTD; 2007.

17. Organización Mundial de la Salud (OMS). Cuestionario de Identificación de los Trastornos Debidos al Consumo de Alcohol. Geneva: OMS; 2001.

18. Reinert DF, Allen JP. The Alcohol Use Disorders Identification Test: An update of research findings. Alcohol Clin Exp Res 2007; 31(2):185-199.
19. Cohen S, Kamarck T, Mermelstein. A Global Measure of Perceived Stress. Journal of Health and Social Behavior. 1983; 24(4):385-396.

20. González MT, Landero R. Factor Structure of the Perceived Stress Scale (PSS) in a Sample from Mexico. Span J Psychol 2007; 10(1):199-206.

21. Ellison N, Steinfield C, Lampe C. The benefits of Facebook "friends:" social capital and collage students' use of online social network sites. Journal of Computer-Mediated Communication 2007; 12(4):1143-1168.

22. Forest A, Wood J. When social networking is not working: individuals with low self-esteem recognize but do not reap the benefits of self-disclosure on Facebok. Psychol Sci 2012; 23(3):295-302.

23. Skues J, Williams B, Wise L. The effects of personality traits, self-esteem, loneliness, and narcissism on Facebook use among university students. Computers in $\mathrm{Hu}$ man Behavior 2012; 28(6):2414-2419.

24. Pettjohn T, LaPiene K, Pettjohn T, Horting A. Relationships between Facebook intensity, friendship contingent self-esteem, and personality in U.S. collage students. Cyberpsychology: Journal of Psychosocial Research on Cyberspace 2012; 6(1):article 2.

25. Moreno M, Grant A, Kacvinsky L, Egan K, Fleming M. Collage students' alcohol displays on Facebook: intervention considerations. J Am Coll Health 2012; 60(5):388-394.

26. Glassman T. Implications for Collage Students Posting Picture of Themselves Drinking Alcohol on Facebook. Journal of Alcohol \& Drug education 2012; 56(1):38-58.

27. Fogg BJ, Eckles D. Mobile Persuasion: 20 Perspectives on the Future of Behavior Change. Stanford: Stanford Captology Media; 2008.

28. Kimbrough AM, Guadagno RE, Muscanell NL, Dill J. Gender differences in mediated communication: Women connect more than do men. Computers in $\mathrm{Hu}$ man Behavior 2013; 29(3):896-900.

29. Guadagno R E, Cialdini RB. Online persuasion: An examination of gender differences in computer-mediated interpersonal influence. Group Dynamics: Theory Research and Practice 2002; 6:38-51.

30. Fardouly J, Diedrichs PC, Vartanian L, Halliwell E. Social comparisons on social media: The impact of Facebook on young womens body image concerns and mood. Body Image 2010; 13:38-45.

31. Diener E, Seligman MEP. Very happy people. Psychological Science 2002; 13(1):80-83.

32. Mehl MR, Vazire S, Holleran SE, Clark CS. Eavesdropping on Happiness: Well-being is Related to Having Less Small Talk and More Substantive Conversations. Psychological Science 2010; 21(4):539-541.

Artigo apresentado em 31/05/2016

Aprovado em 07/11/2016

Versão final apresentada em 09/11/2016 
